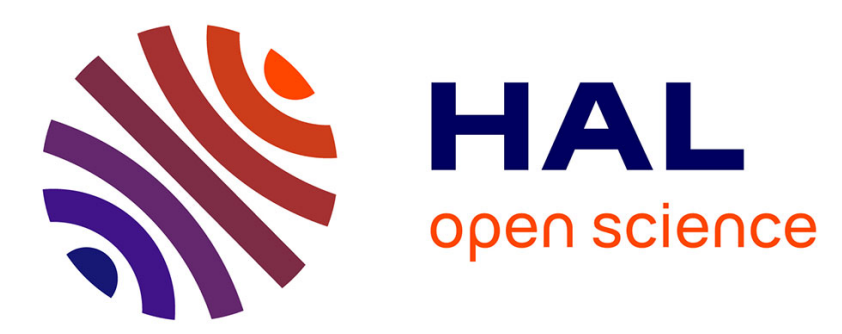

\title{
Effects of the V82A and I54V mutations on the dynamics and ligand binding properties of HIV-1 protease
}

Pia Dirauf, Heike Meiselbach, Heinrich Sticht

\section{- To cite this version:}

Pia Dirauf, Heike Meiselbach, Heinrich Sticht. Effects of the V82A and I54V mutations on the dynamics and ligand binding properties of HIV-1 protease. Journal of Molecular Modeling, 2010, 16 (10), pp.1577-1583. 10.1007/s00894-010-0677-9 . hal-00570125

\section{HAL Id: hal-00570125 \\ https://hal.science/hal-00570125}

Submitted on 27 Feb 2011

HAL is a multi-disciplinary open access archive for the deposit and dissemination of scientific research documents, whether they are published or not. The documents may come from teaching and research institutions in France or abroad, or from public or private research centers.
L'archive ouverte pluridisciplinaire HAL, est destinée au dépôt et à la diffusion de documents scientifiques de niveau recherche, publiés ou non, émanant des établissements d'enseignement et de recherche français ou étrangers, des laboratoires publics ou privés. 


\section{Editorial Manager(tm) for Journal of Molecular Modeling Manuscript Draft}

Manuscript Number: JMM01146

Title: Effects of the V82A and I54V mutations on the dynamics and ligand binding properties of HIV-1 Protease

Article Type: Original paper

Keywords: HIV-1 protease; V82A mutant; molecular dynamics; drug resistance; amprenavir; ritonavir

Corresponding Author: Prof. Heinrich Sticht,

Corresponding Author's Institution:

First Author: Pia Dirauf

Order of Authors: Pia Dirauf; Heike Meiselbach; Heinrich Sticht

Abstract: A major problem in the antiretroviral treatment of HIV-infections with protease-inhibitors is the emergence of resistance, resulting from the occurrence of distinct mutations within the protease molecule. In the present work we investigated the structural properties of a triple mutant (I54V-V82AL90M) and a double mutant (V82A-L90M) that both confer strong resistance to ritonavir (RTV), but not to amprenavir (APV).

For the unliganded double mutant protease molecular dynamics simulations revealed a contraction of the ligand binding pocket, which is enhanced by the I54V mutation. The observed displacement of backbone atoms of the 80s loops (residues 80-85 and 80'-85' of the dimer) was found to primarily affect binding of the larger RTV molecule. The pocket contraction detected for the unbound protease upon mutation is also observed in the presence of APV, but not of RTV. As a consequence, the proteinligand contacts lost upon the V82A mutation are restored by 80s loop motions for the APV-bound, but not for the RTV-bound form.

RTV binding is therefore both hampered in the initial recognition step due to the poor fit of the bulky inhibitor into the small pocket of the mutant free protease and by the loss of protein-ligand interactions in the RTV-bound protease. The synergistic nature of both effects offers an explanation for the high level of resistance observed.

These findings demonstrate that large inhibitors, which tightly bind to wild-type protease, may nevertheless be prone to the emergence of resistance in the presence of particular patterns of mutations. This information should be helpful for the design of novel and more effective drugs, e.g. by targeting different residues or by developing allosteric inhibitors that are capable of regulating protease dynamics. 


\title{
Effects of the V82A and I54V mutations on the dynamics and ligand binding properties of HIV-1 protease
}

Received: 08.12.2009 / Accepted: 02.02.2010

Pia Dirauf $^{1}$, Heike Meiselbach ${ }^{1}$, and Heinrich Sticht ${ }^{1 \bowtie}$

${ }^{1}$ Bioinformatik, Institut für Biochemie, Emil-Fischer-Zentrum, and

Friedrich-Alexander-Universität Erlangen-Nürnberg, 91054 Erlangen, Germany

${ }^{\circledR}$ Tel.:+49 - 9131 / 85 24614; Fax:+49 - 9131 / 85 22485; E-Mail: h.sticht@ biochem.unierlangen.de

\begin{abstract}
A major problem in the antiretroviral treatment of HIV-infections with protease-inhibitors is the emergence of resistance, resulting from the occurrence of distinct mutations within the protease molecule. In the present work we investigated the structural properties of a triple mutant (I54V-V82A-L90M) and a double mutant (V82A-L90M) that both confer strong resistance to ritonavir (RTV), but not to amprenavir (APV).

For the unliganded double mutant protease molecular dynamics simulations revealed a contraction of the ligand binding pocket, which is enhanced by the I54V mutation. The observed displacement of backbone atoms of the 80s loops (residues 80-85 and 80'-85' of the dimer) was found to primarily affect binding of the larger RTV molecule. The pocket contraction detected for the unbound protease upon mutation is also observed in the presence of APV, but not of RTV. As a consequence, the protein-ligand contacts lost upon the V82A mutation are restored by 80 s loop motions for the APV-bound, but not for the RTV-bound form.
\end{abstract}

RTV binding is therefore both hampered in the initial recognition step due to the poor fit of the bulky inhibitor into the small pocket of the mutant free protease and by the loss of proteinligand interactions in the RTV-bound protease. The synergistic nature of both effects offers an explanation for the high level of resistance observed.

These findings demonstrate that large inhibitors, which tightly bind to wild-type protease, may nevertheless be prone to the emergence of resistance in the presence of particular patterns of mutations. This information should be helpful for the design of novel and more 
effective drugs, e.g. by targeting different residues or by developing allosteric inhibitors that are capable of regulating protease dynamics.

Keywords HIV-1 protease - V82A mutant - Molecular dynamics - Drug resistance • Amprenavir · Ritonavir 


\section{Introduction}

The retrovirus Human Immunodeficiency Virus Type-1 (HIV-1) is the causing agent of the acquired immunodeficiency syndrome (AIDS) [1]. The retroviral aspartic protease of HIV-1 (Fig. 1A) plays an essential role in the viral replication cycle, and inhibition of HIV-1 protease has been shown to result in the production of non-infectious virions [2]. The enzyme is composed as a homodimer of two peptide chains with 99 amino acids each, and its active site contains two aspartic acid residues, one contributed by each subunit [3].

In AIDS therapy approaches, HIV-1 protease has very early been recognized as a target protein and numerous protease inhibitors are in clinical use today. However, the rapid emergence of resistant and cross-resistant strains by mutation of the protease gene poses a great challenge to all efforts of providing effective treatment. Although databases like the Stanford HIV Database [4] offer extensive statistical data about genotype-phenotype correlations, still little is known about the actual resistance mechanisms of mutations. Especially interactions of multiple mutations are still poorly understood and it is obvious that the effects of single mutations cannot be considered independently, when they occur in combination with amino acid exchanges in other positions of the same target protein.

In the present study, the double mutant V82A-L90M and triple mutant I54V-V82A-L90M were analyzed with a particular focus on the effects of mutations V82A and I54V for conferring differential resistance properties regarding the inhibitors amprenavir (APV) and ritonavir (RTV) (Fig. 1B, 1C).

Both the V82A mutation and the I54V-V82A combination of mutations predominantly occur in the background of a L90M mutation. According to the data of the Stanford University HIV Drug Resistance Database [4], L90M is a very frequent mutation, which itself confers only moderate resistance to RTV and APV. It has also been shown to independently evolve several times in vivo even in the same patient [6]. Therefore, L90M can be considered as genetic background rather than an independent resistance mutation.

The three mutations I54V/V82A/L90M are located in three different functional regions of HIV-1 protease: I54V in the flaps, V82A in the active site and L90M in the dimerization interface (Fig. 1A). The interesting point about the V82A-L90M double and I54V-V82AL90M triple mutant is their strong resistance to RTV and the comparatively weak resistance to APV. The mean fold resistance scores are 14.1 (RTV) vs. 1.6 (APV) for the double mutant 
and 83.0 (RTV) vs. 3.1 (APV) for the triple mutant [4]. The aim of this study was to shed light on the mechanisms that lead to the differential resistance behavior concerning these two protease inhibitors.

\section{Methods and materials}

The starting structures of the protease-drug complexes were taken from the Protein Data Bank (PDB) [7]. The PDB entries were 1HPV for protease complexed with Amprenavir [8] (APV) and 1HXW for the protease with ritonavir (RTV) [9]. There is no high-resolution crystal structure of an unliganded HIV-1 protease available, so the starting structures of the unbound form were generated by deleting the inhibitor from the APV-bound form as already performed in previous work $[10,11]$. Double and triple mutant structures were derived from the unliganded and ligand-bound wild-type structures by insertion of the mutations using SwissPdbViewer [12].

All molecular dynamics (MD) simulations presented in this work were performed using AMBER 9[13-15] with the parm99 force field [16, 17] and the TIP3P water model [18]. For the organic compounds APV and RTV the general AMBER force field (gaff) [19] was used. Missing parameters and partial atomic charges were adopted from a previous quantum mechanical parameterization of APV [10], RTV was parameterized following the same protocol.

Simulations were performed in a periodic water box with at least $10 \AA$ of solvent around every atom of the solute. An appropriate number of counter ions was added to neutralize the charges of the systems, and the Particle Mesh Ewald summation method [20] was employed to calculate the long-range electrostatic interactions. All structures were minimized in a threestep procedure using the SANDER module of AMBER following a previously established protocol $[10,11]$.

MD simulations were performed using the SHAKE procedure [21] to constrain all bonds involving hydrogen atoms. The integration time step of the simulation was 1 fs and an $8.5 \AA$ cutoff was used for the nonbonded interactions which were updated every 15 steps. The temperature of each system was gradually heated to $298 \mathrm{~K}$ during the first $10 \mathrm{ps}$. Subsequently, $20 \mathrm{~ns}$ MD simulations were performed for data collection. 
For the visualization, structural and energetic analysis of the trajectory data the programs Sybyl 7.3 [22], DS ViewerPro Suite 6 [23], and AMBER [14] were used.

\section{Results and discussion}

\section{Analysis of the static complex structures}

Initially, the APV- and RTV-bound protease crystal structures were analyzed to obtain first information about the role of the mutations I54V and V82A for the emergence of differential resistance. V82 is located in the active site and forms direct interactions with APV and RTV. In both complexes, the interactions are exclusively formed by the $\gamma$-methyl groups of the valines suggesting that a mutation to alanine results in a loss of the respective interactions consequently leading to drug resistance. While such an emergence of resistance is observed for RTV (14.1-fold increase) in the V82A-L90M mutant, there is only a moderate increase (1.6-fold) for APV raising the question about putative compensatory mechanisms, which allow APV-binding even in the presence of the V82A active site mutation.

Although I54 is located in the flap region (Fig. 1) and does not form direct contacts to the inhibitor, it nevertheless has a significant and also selective effect on drug resistance. While the resistance to APV is almost unaffected by this mutation, resistance to RTV is significantly increased resulting in an overall 83-fold increased resistance of the I54V-V82A-L90M triple mutant compared to the wild-type. Since these differential effects of the V82A and the I54V mutant on drug resistance cannot be explained from the static structure, we performed molecular dynamics simulations of the ligand-bound and free proteases for wild-type and mutant protein.

\section{Dynamics of the protease binding pocket}

The geometry and dimensions of HIV-1 protease's substrate binding pocket are crucial for binding of competitive inhibitors like APV and RTV. The mutation V82A is located within the 80 s loops (residues $80-85$ and $80^{\prime}-85^{\prime}$ ') of HIV-1 protease that form the lateral surface of the binding pocket (Fig. 1A). The protein conformation in this region is particularly important for the ability of the protease to bind ligands, since local rearrangements will affect the shape 
and size of the binding site. Therefore, pairwise interatomic distances in the protease structure were determined for the $\mathrm{C}_{\alpha}$-atoms of residues that compose the lateral surface of the pocket, namely residues $80 / 80$ ' and $81 / 81^{\prime}$ (Table 1 ).

For the unliganded protease, a decrease of the pocket size ('pocket contraction') was detected in the double mutant, and even to a greater extent in the triple mutant. The $\mathrm{C}_{\alpha}$-atoms of both residue-pairs investigated are about $19 \AA$ apart in the wild-type between 5 and $15 \mathrm{~ns}$ of simulation, while in the triple mutant the distance averages to $16 \AA$ during the same simulation period (Fig. 2A, Table 1). The effect is also seen with the double mutant, but less pronounced. Although there is a transient increase of the distances towards the end of the simulation in the triple mutant, the averaged distances over the entire simulation time are still considerably smaller than the corresponding values in the wild-type and double mutant structures (Table 1). These findings indicate that the protein backbone atoms of the 80s loops move towards each other in the double mutant, and that this effect is enhanced by the additional mutation I54V. The fact that the decreased distance between the 80s loops is observed in the unliganded protease indicates that this effect can be attributed to the mutations themselves and occurs independently from ligand binding. This observation suggests that the pocket of the mutant protease might become too small to accommodate the inhibitors thereby leading to resistance.

To substantiate this hypothesis, the spatial requirement for inhibitor binding was determined by measuring the pocket size in the simulations of APV- and RTV-bound protease (Table 1). For the APV-bound protease, the distances are generally quite similar to those observed in the unliganded protease (Table 1). Therefore, the smaller pocket observed for the double and triple mutant is expected to pose no severe problem on APV binding. In addition, it is interesting to note that the ability of pocket contraction, which was observed for the unliganded protease, is also retained for the APV-bound form. The extent of these motions of the 80s loops is shown in detail in Fig. 3. Similar backbone displacements of the 80s loops have also been found in previous MD studies with inhibitor-bound V82F/I84V double mutant [24], in X-ray diffraction studies of a V82A mutant in complex with other inhibitors [25, 26], as well as for the single mutation I54V [27].

In contrast, for the RTV-bound protease, the distances between the 80s loops are generally large and are almost unaffected by the pattern of mutations (Table 1). This observation can be explained by the fact that RTV is a bulky inhibitor (Fig. 1C) which requires a large binding 
pocket and consequently also blocks motions of the 80s loops which would lead to a smaller binding pocket.

Comparison of the space required for RTV binding to the size of the pocket in the unliganded protease also suggests a structural mechanism contributing to RTV resistance: In the free wild-type protease, the pocket is sufficiently large to accommodate RTV but the smaller size of the pocket in the double and in particular in the triple mutant is expected to hamper RTV binding, The difference between RTV space requirement and the size of the empty binding pocket, measured by the average $81 / 81^{\prime} \mathrm{C}_{\alpha}$-distance in the triple mutant, is as large as $2.25 \AA$ (Table 1A/B, third line). The difference between RTV space requirement and the size of the empty binding pocket, measured by the average $81 / 81^{\prime} \mathrm{C}_{\alpha}$-distance in the triple mutant, is as large as $2.25 \AA$ (Table 1 , last row).

The consequences of these differences in size are emphasized by molecular modeling: Generation of a hybrid complex by placing RTV into the pocket of the unliganded I54VV82A-L90M protease reveals clashes between the two aromatic rings of RTV and the two proline residues in positions 81 and 81 '. In subunit $A$ the $C \gamma$ atom of residue $\mathrm{P} 81$ causes the clash with RTV, while in subunit B it is the $\beta$ atom of $\mathrm{P} 81^{\prime}$ that interferes with inhibitor binding (Fig. 4). The rigid ring structure of P81 impedes a rotation of the proline sidechain that would be necessary to accommodate RTV. Therefore, RTV as a ligand will require a widening of the pocket upon binding to the free protease, especially for the triple mutant enzyme. Since the respective structural rearrangements are energetically unfavorable, they will reduce RTV binding affinity thereby mediating resistance.

There already exist previous studies in which mutations of HIV-1 protease (N88S, F53L) were shown to affect the conformation of the free protease, rendering it less favorable for ligand binding $[10,28]$. A key property of the pocket contraction observed in the present study is its selective effect on the bulkier inhibitor RTV thus offering a first explanation for differential resistance.

\section{Structural and energetic analysis of protease-ligand interactions}

The studies above showed that pocket contraction upon mutation occurs for APV-bound but not for RTV-bound protease (Table 1). This mechanism is expected to affect not only the initial binding process but also the contacts formed between the inhibitor and the residues 
lining the binding pocket. Therefore, a more detailed structural and energetic analysis of the protein-ligand interactions was performed.

The differences between APV-bound and RTV-bound protease become particularly evident for the site of the V82A mutation itself. The $\gamma$-methyl group of V82 forms numerous contacts with APV and RTV in the wild-type protease (Fig. 5). Pocket contraction, which is observed only for the APV-bound protease, allows the A82 sidechain to form contacts to the inhibitor (Fig. 5B). In contrast, motions of the 80s loops are not possible for the bulkier RTV and therefore the loss of V82-ligand contacts cannot be restored by the shorter A82 sidechain (Fig. $5 \mathrm{~A})$.

The strength of the contacts observed between residue 82 and the inhibitors APV and RTV is also reflected in the energetic analysis (Fig. 6). The V82A mutation leads only to a moderate decrease of the interaction energy with APV of approximately $0.5 \mathrm{kcal} \cdot \mathrm{mol}^{-1}$ (Fig. 6A, Table 2). It is also interesting to note that the triple mutant is more effective in restoring wild-type like interaction energies than the double mutant. The most likely explanation comes from the observation that the pocket contraction is larger in the triple mutant compared to the double mutant (Fig. 2, Table 1), which allows tighter contacts between A82 and APV.

Due to the absence of a pocket contraction mechanism, the loss of interaction energy upon V82A mutation is larger for RTV compared to APV. Interaction energies with RTV are strongly reduced in both, double and triple mutants (Table 2). With a loss of approximately $1.2 \mathrm{kcal} \mathrm{mol}^{-1}$ compared to the wild-type, the effect is most pronounced for subunit 2 (Table 2, last row). The unfavorable effect on the interaction energies appears to be caused primarily by mutation V82A, as addition of mutation I54V does not cause any additional significant effects.

The latter finding is in apparent contradiction to the experimental data that the triple mutant shows a significantly enhanced RTV-resistance compared to the double mutant (83-fold vs. 14-fold resistance). The experimental data, however, can be explained by additionally taking into account the pocket contraction observed for the unbound protease. Pocket contraction is more pronounced for the triple compared to the double mutant (Fig. 2, Table 1) suggesting that binding of the bulky RTV will be more severely hindered to the triple mutant. 


\section{Conclusions}

In summary, the present study reveals two effects, which lead to the selective RTV resistance of the V82A-L90M double mutant and the I54V-V82A-L90M triple mutant: Firstly, the initial binding of the bulky inhibitor RTV is hindered by the smaller pocket present in the mutant unliganded protease. Secondly, no pocket contraction is possible in the presence of RTV, which hinders the shorter sidechain of A82 to compensate for the contacts formed between V82 and RTV in the wild-type protease. This effect is expected to enhance the first mechanism thereby explaining the high overall level of resistance.

The present study also shows that large inhibitors, which tightly bind to wild-type protease, may nevertheless be prone to the emergence of resistance in the presence of particular patterns of mutations. This information should be helpful for the design of novel and more effective drugs, e.g. by targeting different residues or by developing allosteric inhibitors that are capable of regulating protease dynamics.

\section{Acknowledgments}

This work was financially supported by the Johannes und Frieda Marohn-Stiftung. The authors also thank the Regionales Rechenzentrum Erlangen (RRZE) for technical support. 


\section{References}

1. Mitsuya H, Yarchoan R, Broder S (1990) Molecular targets for AIDS therapy. Science 249:1533-1544

2. Kohl NE, Emmi EA, Schleif WA, Davies LJ, Heimbach JC, Dixon RAF (1988) Active human immunodeciency virus protease is required for viral infectivity. Proc Natl Acad Sci USA 85:686-690

3. Meek TD, Dayton BD, Metcalf BW, Dreyer GB, Strickler JE, Gorniak JG, Rosenberg M, Moore ML, Magaard VW, Debouck C (1989) Human immunodeficiency virus 1 protease expressed in Escherichia coli behaves as a dimeric aspartic protease. Proc Natl Acad Sci USA 86(6):1841-1845

4. Shafer RW (2006) Rationale and Uses of a Public HIV Drug-Resistance Database. Journal of Infectious Diseases 194 Suppl 1:S51-8. Stanford HIV Database. http://hivdb.stanford.edu. Accessed 3 December 2009

5. Kanehisha Laboratories, Institute for Chemical Research, Kyoto University (2009) KEGG Release 52.1. http://www.genome.jp/kegg/drug. Accessed 3 December 2009

6. Kapoor A, Shapiro B, Shafer RW, Shulman N, Rhee SY, Delwart EL (2008) Multiple independent origins of a protease inhibitor resistance mutation in salvage therapy patients. Retrovirology 5:7

7. Berman HM, Westbrook J, Feng Z, Gilliland G, Bhat TN, Weissig H, Shindyalov IN, Bourne PE (2000) The Protein Data Bank. Nucleic Acids Res 28(1):235-242

8. Kim EE, Baker CT, Dwyer MD, Murcko MA, Rao BG, Tung RD, Navia MA (1995) Crystal Structure of HIV-1 Protease in Complex with VX-478, a potent and orally bioavailable Inhibitor of the Enzyme. J Am Chem Soc 117:1181-1182

9. Kempf DJ, Marsh KC, Denissen JF, McDonald E, Vasavanonda S, Flentge CA, Green BE, Fino L, Park CH, Kong XP et al. (1995) ABT-538 is a potent inhibitor of human immunodeficiency virus protease and has high oral bioavailability in humans. Proc Natl Acad Sci USA 92(7):2484-2488

10. Wartha F, Horn AHC, Meiselbach H, Sticht H (2005) Molecular Dynamics Simulations of HIV-1 Protease Suggest Different Mechanisms Contributing to Drug Resistance. J Chem Theory Comput1:315-324

11. Meiselbach H, Horn AH, Harrer T, Sticht H (2007) Insights into amprenavir resistance in E35D HIV-1 protease mutation from molecular dynamics and binding free-energy calculations. J Mol Model 13(2):297-304 
12. Guex N, Peitsch MC (1997) SWISS-MODEL and the Swiss-Pdb Viewer: An environment for comparative protein modeling. Electrophoresis 18:2714-2723

13. Case DA, Pearlman DA, Caldwell JW, III TEC, Wang J, Ross WS, Simmerling CL, Darden TA, Merz KM, Stanton RV, Cheng AL, Vincent JJ, Crowley M, Tsui V, Gohlke H, Radmer RJ, Duan Y, Pitera J, Massova I, Seibel GL, Singh UC, Weiner PK, Kollman PA (2007) AMBER7. University of California, San Francisco

14. Pearlman DA, Case DA, Caldwell JW, Ross WS, Cheatham TE, 3rd, DeBolt S, Ferguson D, Seibel G, Kollman P (1995) AMBER, a package of computer programs for applying molecular mechanics, normal mode analysis, molecular dynamics and free energy calculations to simulate the structural and energetic properties of molecules. Comput Phys Commun 91:1-41

15. Case DA, Darden TA, Cheatham TE, III, Simmerling CL, Wang J, Duke RE, Luo R, KMerz M, Pearlman DA, Crowley M, Walker RC, Zhang W, Wang B, Hayik S, Roitberg A, Seabra G, Wong KF, Paesani F, Wu X, Brozell S, Tsui V, Gohlke H, Yang L, Tan C, Mongan J, Hornak V, Cui G, Beroza P, Mathews DH, Schafmeister C, Ross WS, Kollman PA (2006) AMBER 9. University of California, San Francisco

16. Cheatham TE, Cieplak P, Kollman PA (1999) A modified version of the Cornell et al. force field with improved sugar pucker phases and helical repeat. J Biomol Struct Dyn 16(4):845-862

17. Cornell WD, Cieplak P, Bayly CI, Gould IR, Merz KMJ, Ferguson DM, Spellmeyer DC, Fox T, Caldwell JW, Kollman PA (1995) A Second Generation Force Field for the Simulaton of Proteins, Nucleic Acids and Organic Molecules. J Am Chem Soc 117:51795197

18. Jorgensen WL, Chandrasekhar J, Madura JD, Impey RW, Klein ML (1983) Comparison of simple potential functions for simulating liquid water. J Chem Phys 79:926-935

19. Wang J, Wolf RM, Caldwell JW, Kollman PA, Case DA (2004) Development and testing of a general amber force field. J Comput Chem 25(9):1157-1174

20. Darden TA, York DM, Pedersen LG (1993) Particle mesh Ewald. An N.log(N) method for Ewald sums in large systems. J Chem Phys 98:10089-10092

21. Ryckaert JP, Ciccotti G, Berendsen HJC (1977) Numerical integration of the Cartesian equations of motion of a system with constraints: molecular dynamics of n-alkanes. $\mathrm{J}$ Comput Phys 23:327-341

22. SYBYL 7.3 (2008) Tripos International, 1699 South Hanley Rd., St. Louis, Missouri, 63144, USA 
23. DS ViewerPro Suite. 6.0 (2005) Accelrys Software Inc., San Diego, CA

24. Hou T, Yu R. (2007) Molecular dynamics and free energy studies on the wild-type and double mutant HIV-1 protease complexed with amprenavir and two amprenavir-related inhibitors: mechanism for binding and drug resistance. J Med Chem 50(6):1177-1188

25. Baldwin ET, Bhat TN, Liu B, Pattabiraman N, Erickson JW (1995) Structural basis of drug resistance for the V82A mutant of HIV-1 proteinase. Nat Struct Biol 2(3):244-249

26. Tie Y, Boross PI, Wang YF, Gaddis L, Liu F, Chen X, Tozser J, Harrison RW, Weber IT (2005) Molecular basis for substrate recognition and drug resistance from 1.1 to 1.6 angstroms resolution crystal structures of HIV-1 protease mutants with substrate analogs. FEBS J 272(20):5265-5277

27. Liu F, Kovalevsky AY, Tie Y, Ghosh AK, Harrison RW, Weber IT (2008) Effect of flap mutations on structure of HIV-1 protease and inhibition by saquinavir and darunavir. $\mathbf{J}$ Mol Biol 381(1):102-115

28. Liu F, Kovalevsky AY, Louis JM, Boross PI, Wang YF, Harrison RW, Weber IT (2006) Mechanism of drug resistance revealed by the crystal structure of the unliganded HIV-1 protease with F53L mutation. J Mol Biol 358(5):1191-1199 


\section{Tables}

Table 1 Mean interatomic distances [Å] between $\mathrm{C}_{\alpha^{-}}$-atoms of residues T80 and T80' (A), and residues $\mathrm{P} 81$ and $\mathrm{P} 81$ ' (B) over the course of simulation

A) average distance between $\mathrm{T} 80\left(\mathrm{C}_{\alpha}\right)$ and $\mathrm{T} 80^{\prime}\left(\mathrm{C}_{\alpha}\right)$

\begin{tabular}{llll} 
& unliganded protease & protease with RTV & protease with APV \\
\hline wild type & 19.12 & 19.07 & 19.19 \\
V82A-L90M & 18.23 & 19.92 & 19.29 \\
I54V-V82A-L90M & 17.89 & 19.05 & 17.90
\end{tabular}

B) average distance between $\mathrm{P} 81\left(\mathrm{C}_{\alpha}\right)$ and $\mathrm{P} 81^{\prime}\left(\mathrm{C}_{\alpha}\right)$

\begin{tabular}{llll} 
& unliganded protease & protease with RTV & protease with APV \\
\hline wild type & 19.22 & 20.41 & 19.90 \\
V82A-L90M & 19.14 & 19.86 & 19.86 \\
I54V-V82A-L90M & 18.54 & 20.79 & 18.44
\end{tabular}


Table 2 Mean values of van der Waals interaction energies of the residues in sequence positions 82 with APV (A) and RTV (B) over the course of simulation

A) van der Waals interaction energies of HIV-1 protease residues 82 and 82'with APV

\begin{tabular}{llll} 
residue & $\mathrm{E}_{\text {wild-type }}\left[\mathrm{kcal} \cdot \mathrm{mol}^{-1}\right]$ & $\mathrm{E}_{\mathrm{V82A-L90M}}\left[\mathrm{kcal} \cdot \mathrm{mol}^{-1}\right]$ & $\mathrm{E}_{\text {I54V-V82A-L90M }}\left[\mathrm{kcal} \cdot \mathrm{mol}^{-1}\right]$ \\
\hline V82 / A82 & -0.88 & -0.54 & -0.78 \\
$\mathrm{~V} 82^{\prime}$ / A82 & -1.65 & -0.93 & -1.13
\end{tabular}

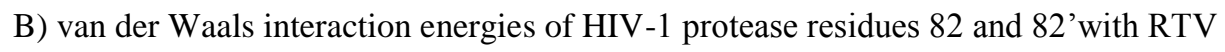

\begin{tabular}{llll} 
residue & $\mathrm{E}_{\text {wild-type }}\left[\mathrm{kcal} \cdot \mathrm{mol}^{-1}\right]$ & $\mathrm{E}_{\mathrm{V82A-L90M}}\left[\mathrm{kcal} \cdot \mathrm{mol}^{-1}\right]$ & $\mathrm{E}_{[54 \mathrm{~V}-\mathrm{V} 82 \mathrm{~A}-\mathrm{L90M}}\left[\mathrm{kcal} \cdot \mathrm{mol}^{-1}\right]$ \\
\hline $\mathrm{V} 82$ / A82 & -1.46 & -0.78 & -0.60 \\
$\mathrm{~V} 82^{\prime}$ / A82 & -2.53 & -1.31 & -1.36
\end{tabular}




\section{Figure captions}

Fig. 1 (A) Schematic view of the dimeric HIV-1 protease and the location of the three mutations studied. The sites of mutations, residues 54 and 54' (magenta), residues 82 and 82' (light blue), residues 90 and 90' (dark blue) are depicted in space-filled representation. Only the backbone atoms of HIV-1 protease are shown, the loops that represent the lateral lining of the ligand binding site (residues 80 to 84 and 80' to 84 ') are colored yellow. (B) amprenavir and (C) ritonavir shown in Fischer's projection [5]

Fig. 2 Interatomic distances between $\mathrm{C}_{\alpha}$-atoms of residues P81 and P81' for unliganded HIV-1 protease (A), protease in complex with APV (B), and protease in complex with RTV (C). Wild-type colored grey, double mutant (V82A-L90M) blue, triple mutant (I54V-V82A-L90M) magenta

Fig. 3 Overlay of APV-bound wild-type (grey) and triple mutant (I54V-V82A-L90M, red) protease 80s loops after $15 \mathrm{~ns}$ of simulation. Protein backbone shown in $\mathrm{C}_{\alpha}$-stick representation, surface of wild-type (grey) and triple mutant (red) depicted semitransparent

Fig. 4 Clashes between residues P81 and P81' and RTV as observed when placing RTV into the contracted binding pocket of the triple mutant (I54V-V82A-L90M) unliganded protease. Shown here is a modeled hybrid complex of RTV placed into the conformation of the unliganded triple mutant protease after $15 \mathrm{~ns}$ of simulation. RTV is shown in surface representation in standard CPK coloring. Residues P81 and P81' are shown in orange with wire mesh surface, residues A82 and A82' are shown in blue with wire mesh surface. Backbone atoms of other residues are depicted as a grey trace

Fig. 5 Overlay of protease structures with RTV (A) and APV (B), showing residues 81, 81', 82 and 82' after 7 ns of simulation. Wild-type shown in grey, triple mutant (I54V-V82A-L90M) in red 
Fig. 6 Van der Waals interaction energies of residues 82 and 82' with APV (A) and RTV (B) as a function of simulation time. Wild-type shown in grey, double mutant (V82A-L90M) in blue, triple mutant (I54V-V82A-L90M) in magenta. Interaction energy of A) residue 82 with APV (left), residue 82' with APV (right), B) residue 82 with RTV (left), residue 82 ' with RTV (right). Since the valine or alanine sidechain at position 82 form only nonpolar contacts to the inhibitor, the van der Waals energy was chosen as a measure of the interaction energy. 


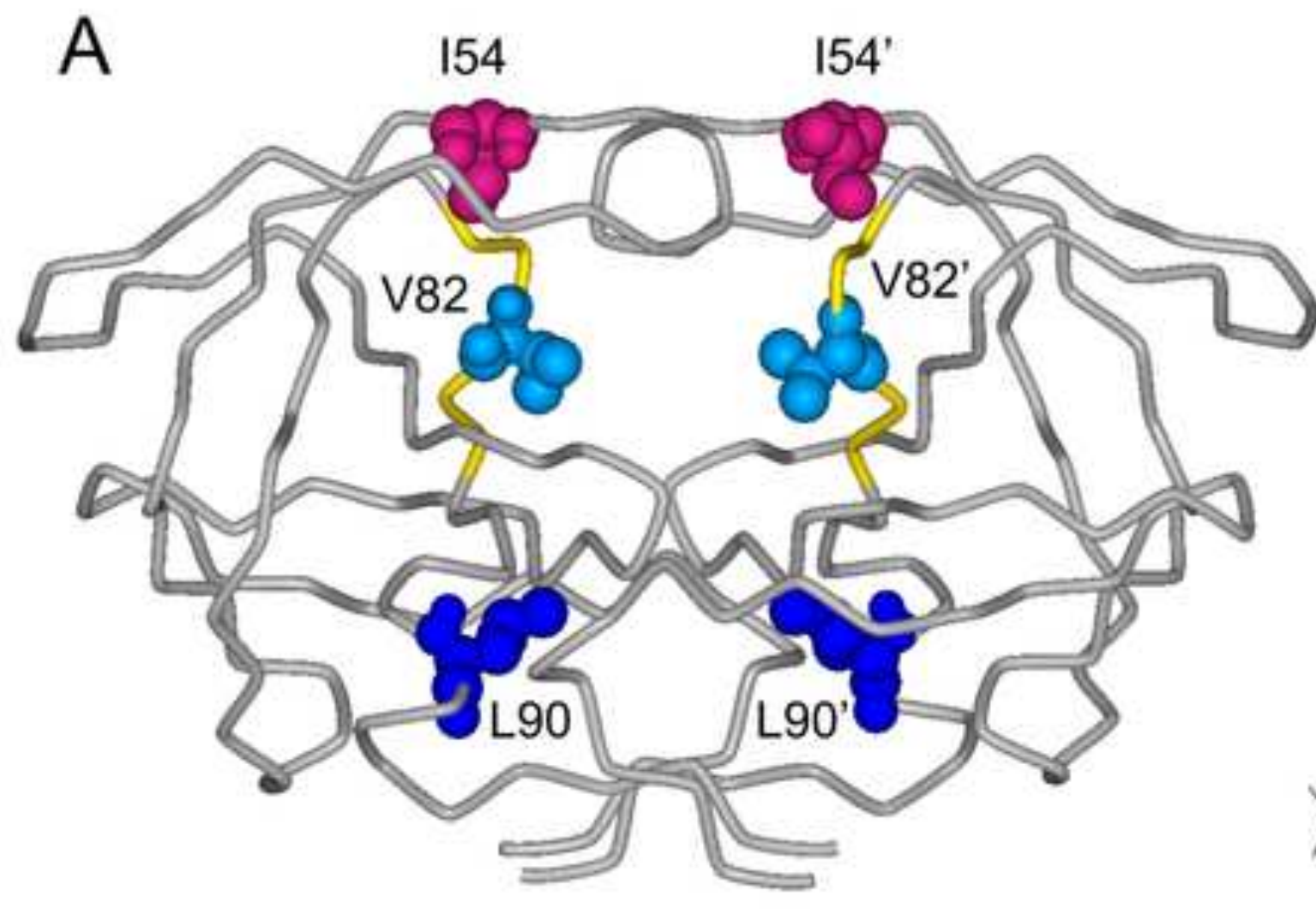

B

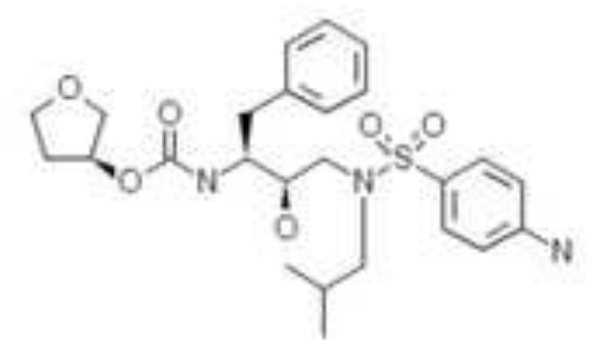

C

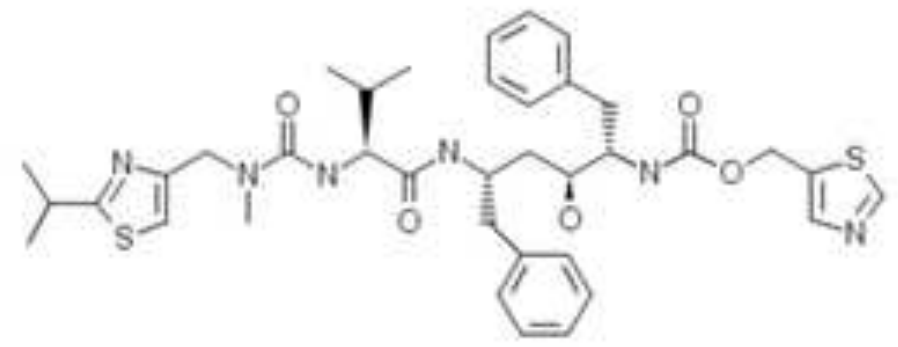


Figure 2

Click here to download high resolution image

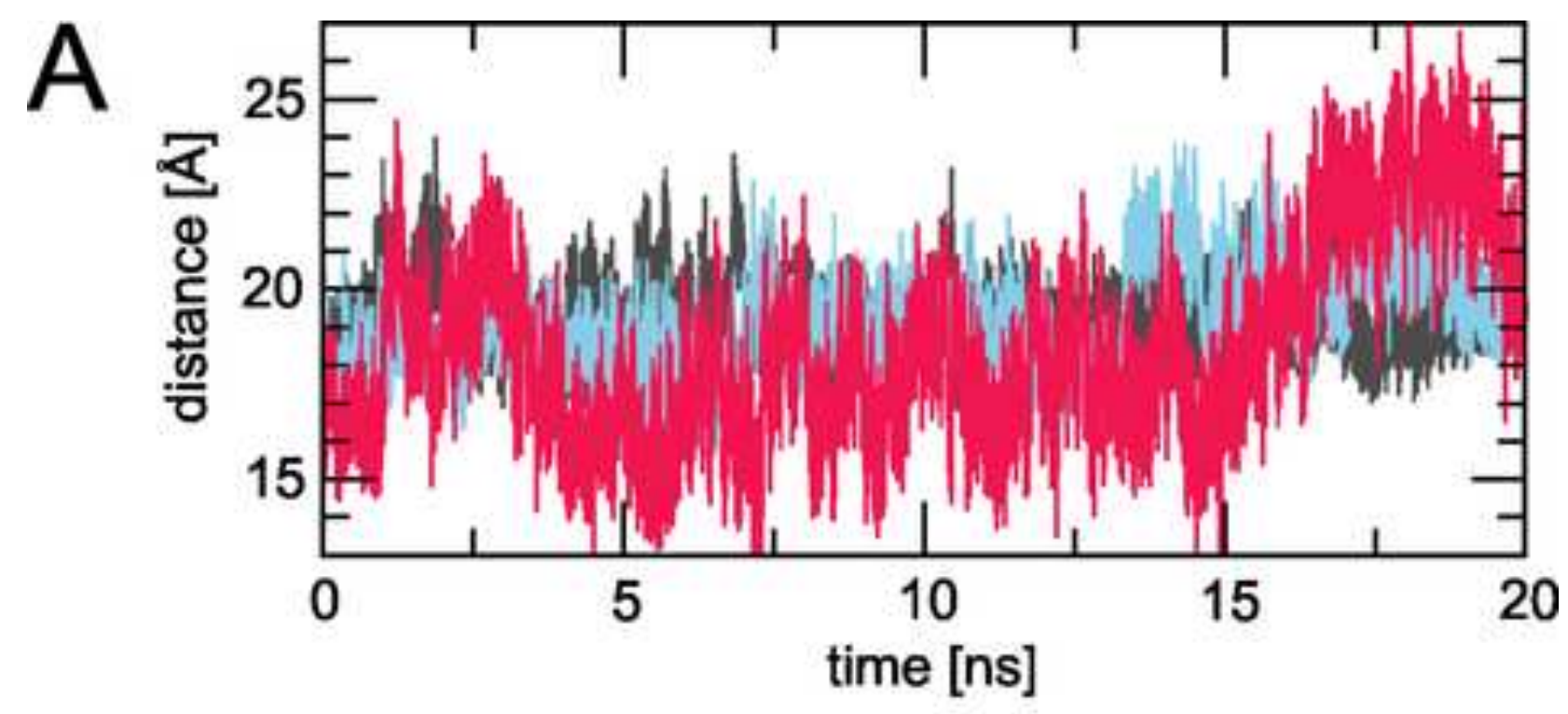

B
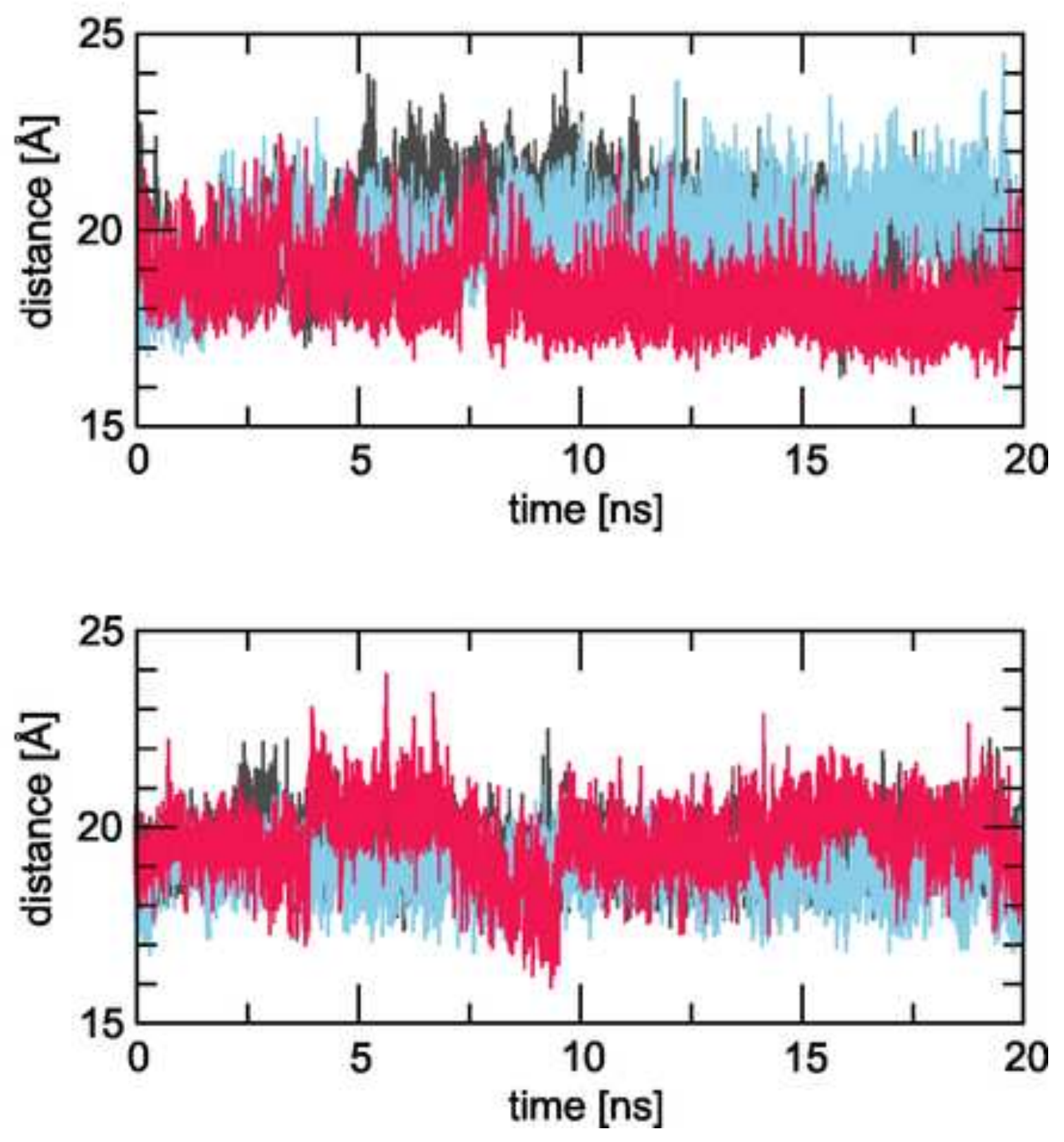
Click here to download high resolution image

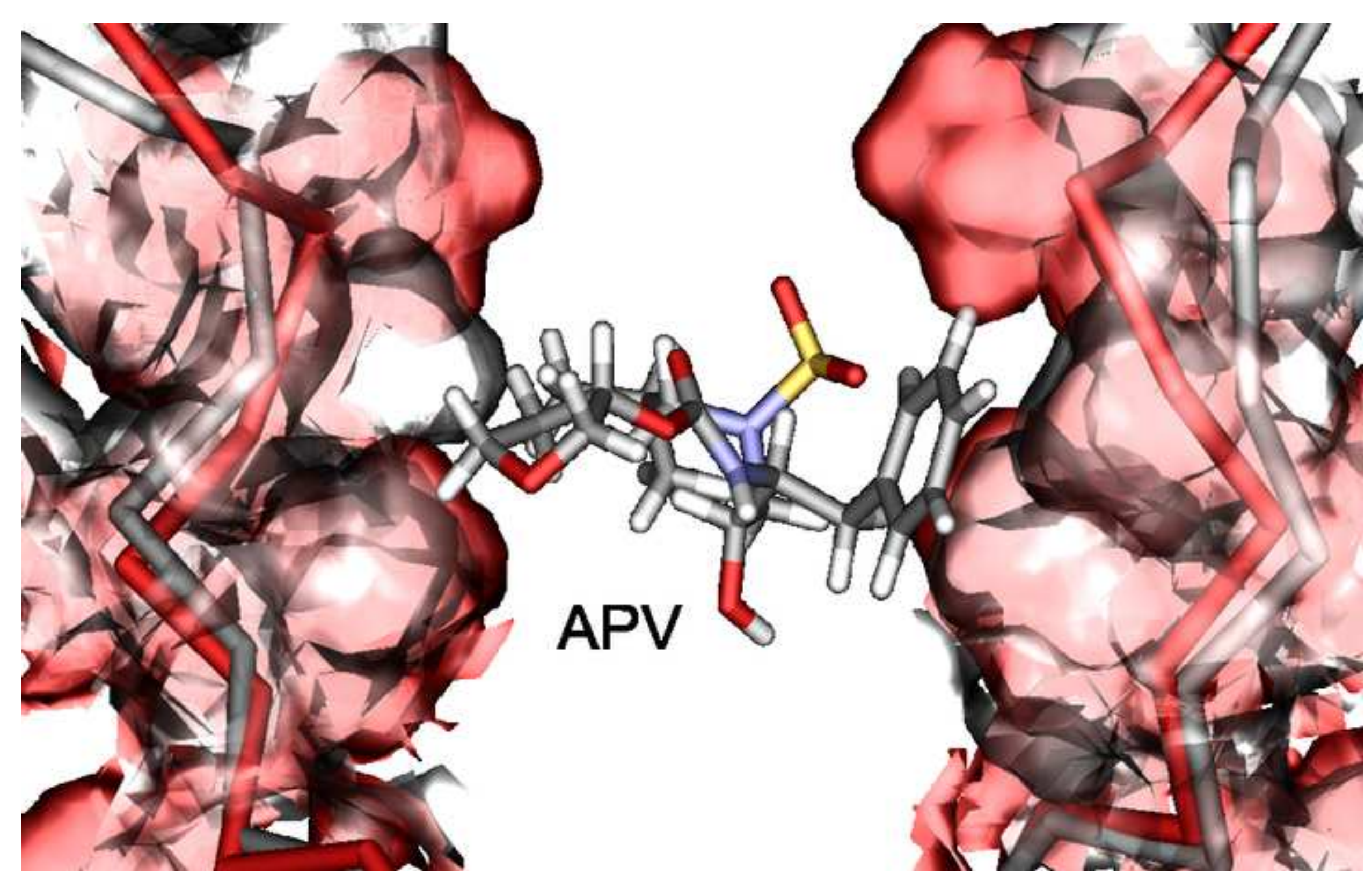

APV $b$

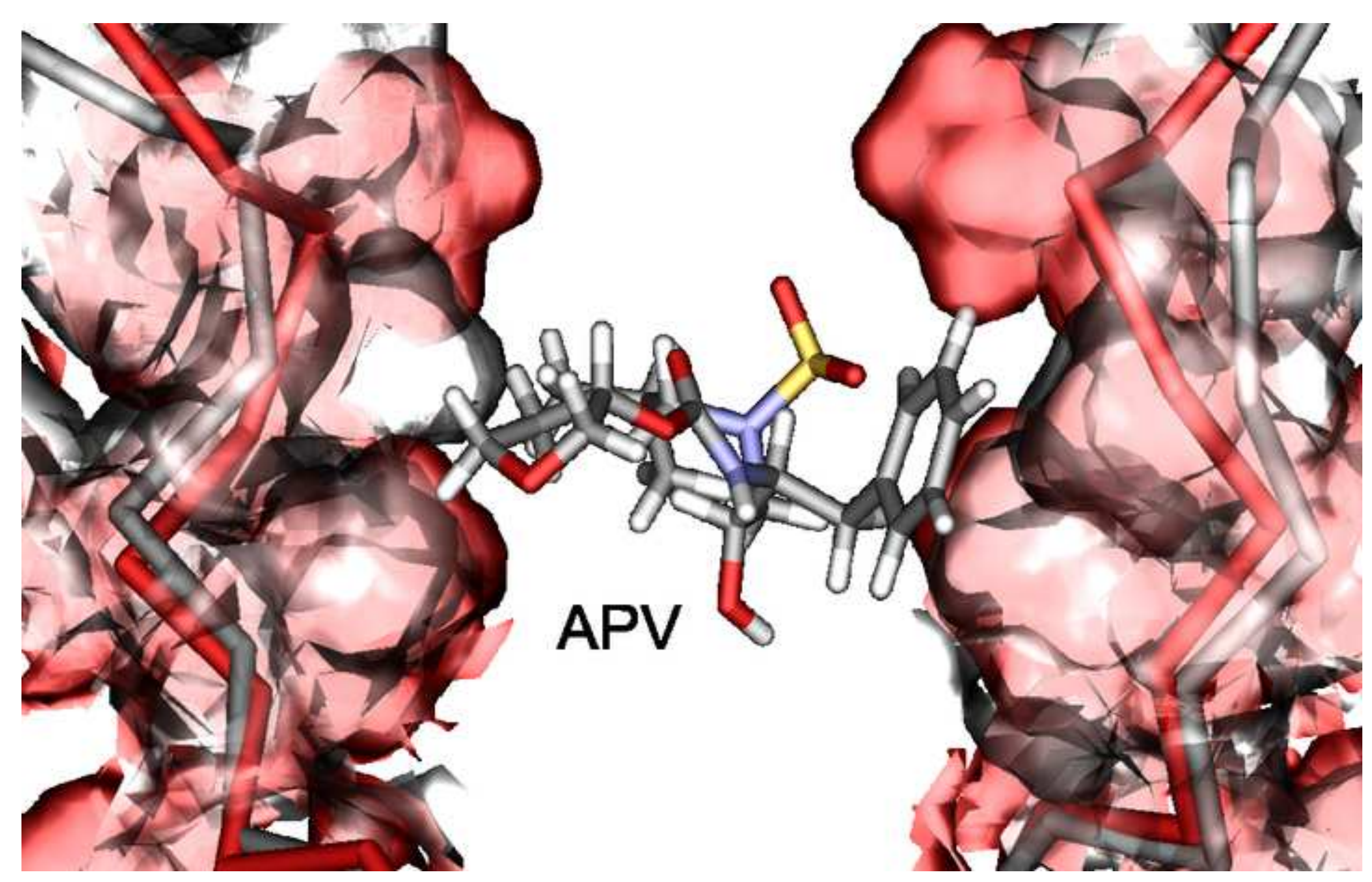


Click here to download high resolution image

\section{P81}

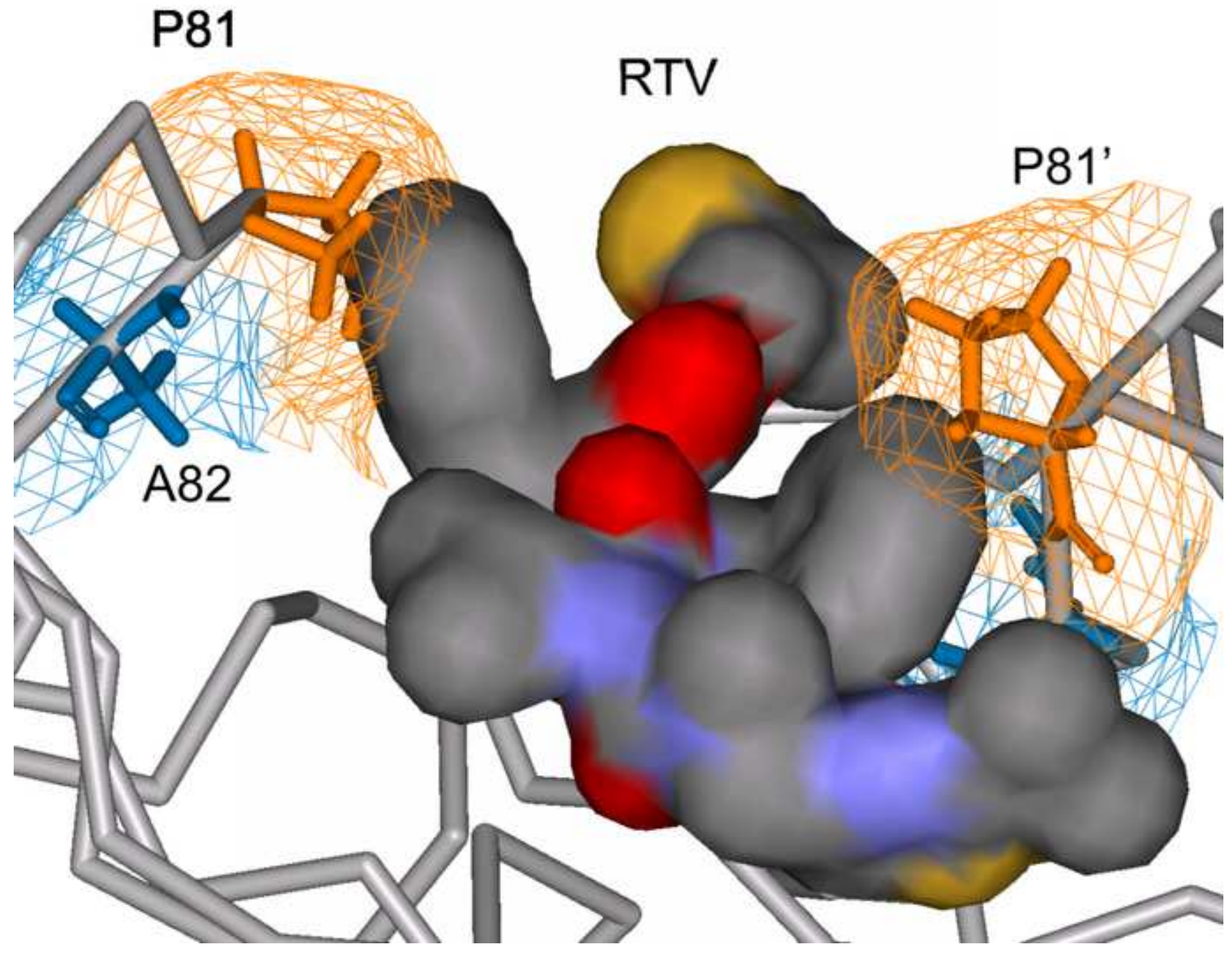


A

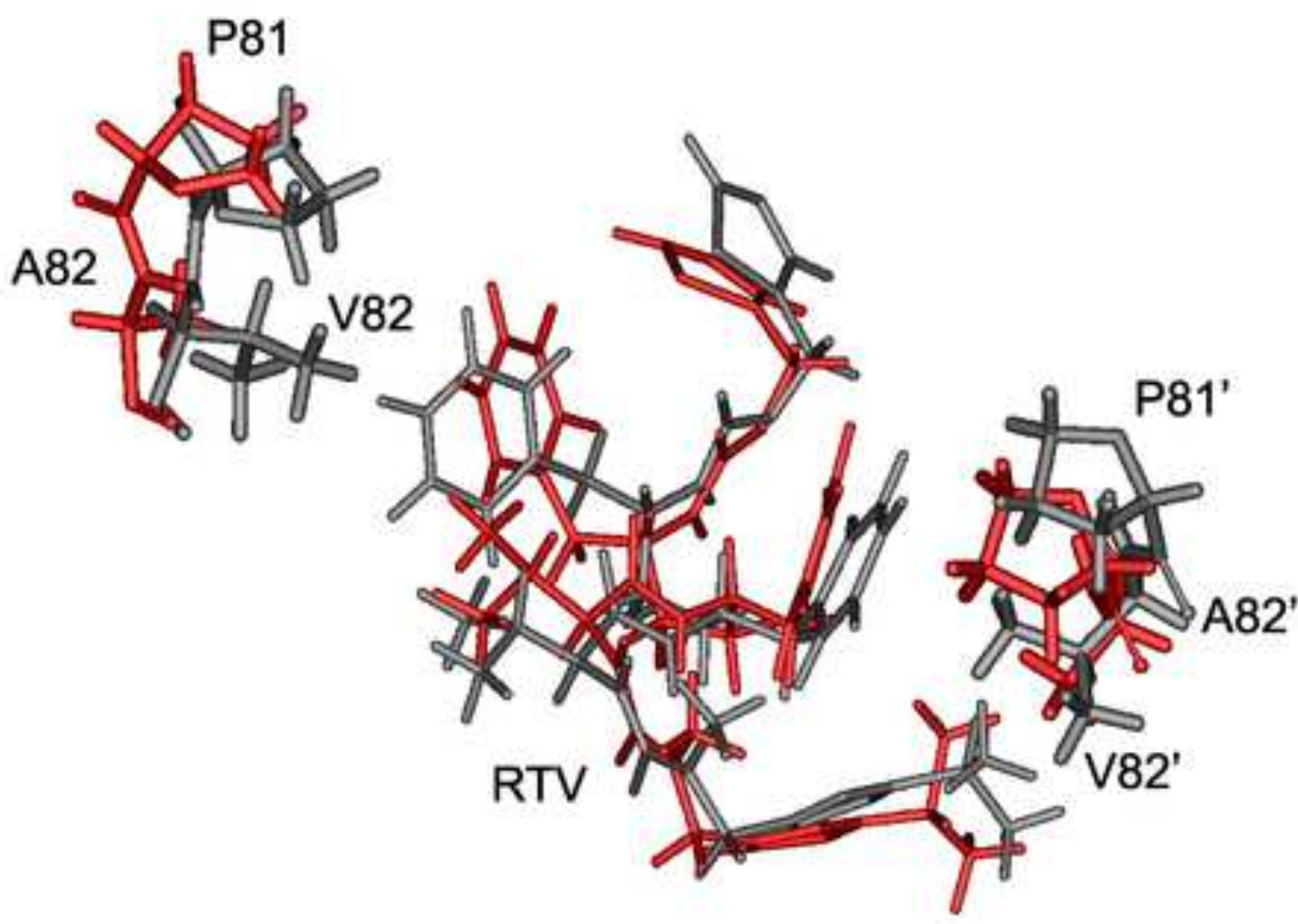

B

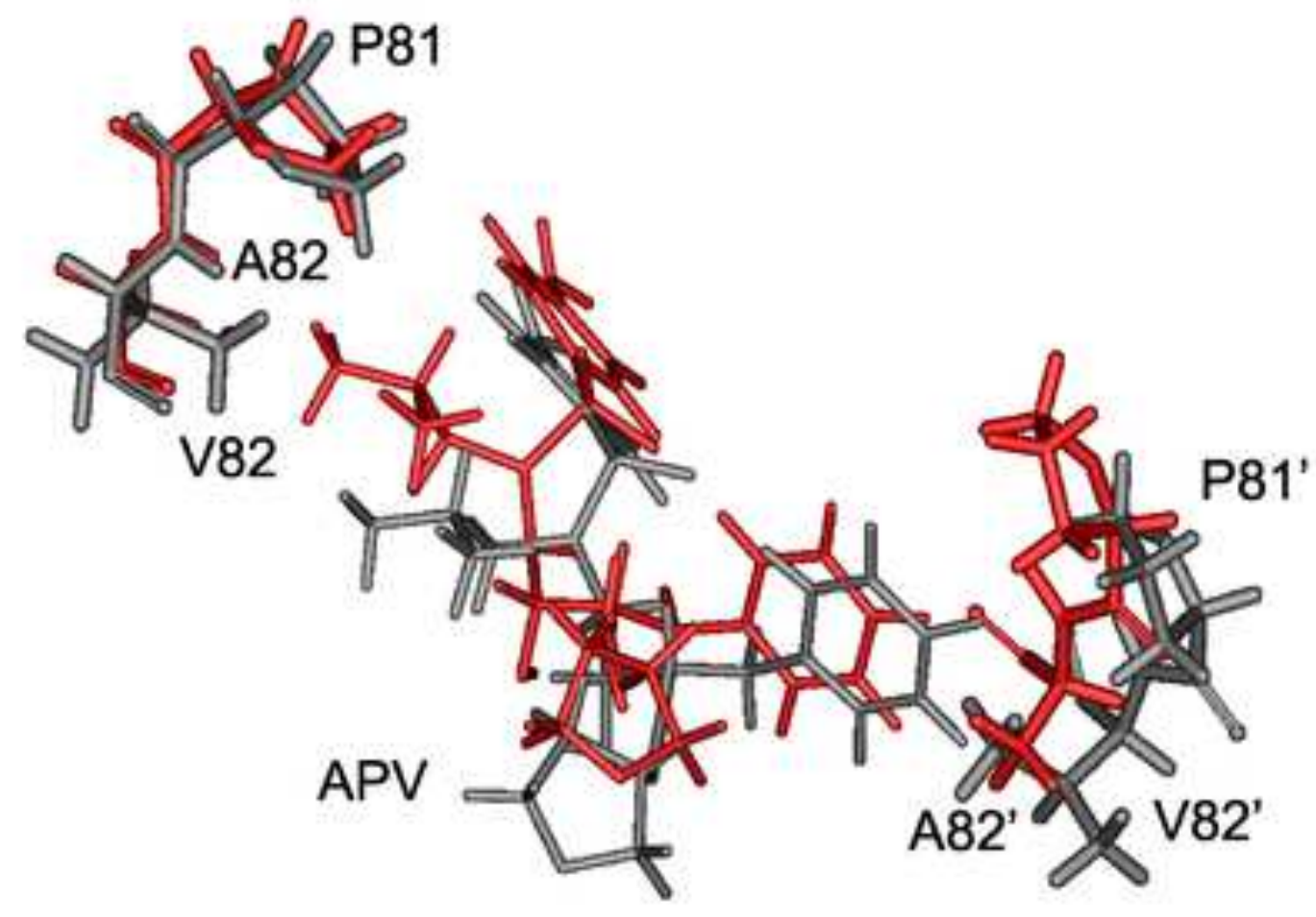


A
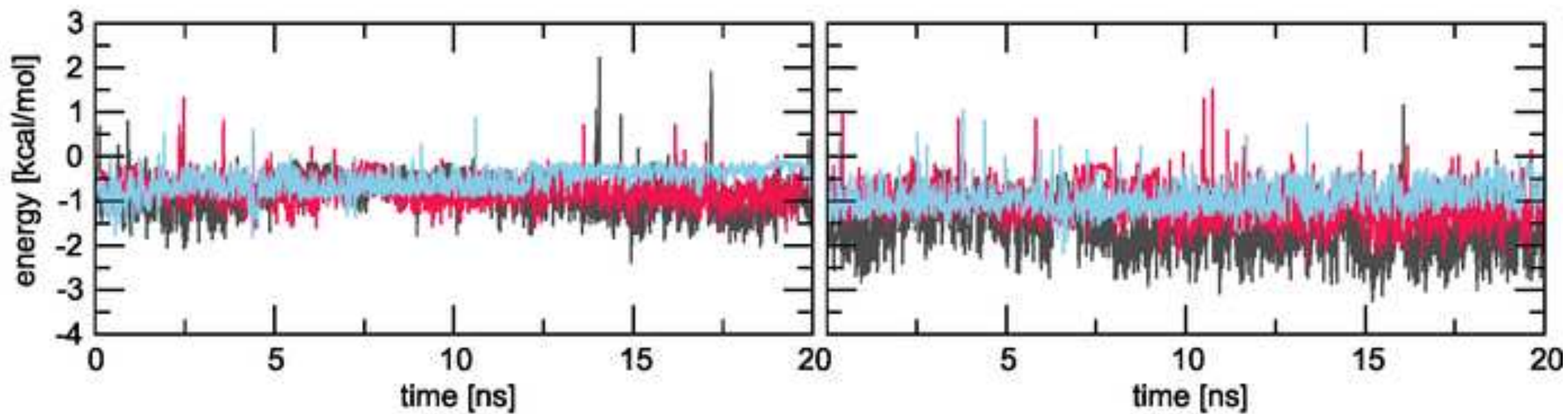

$\mathrm{B}$

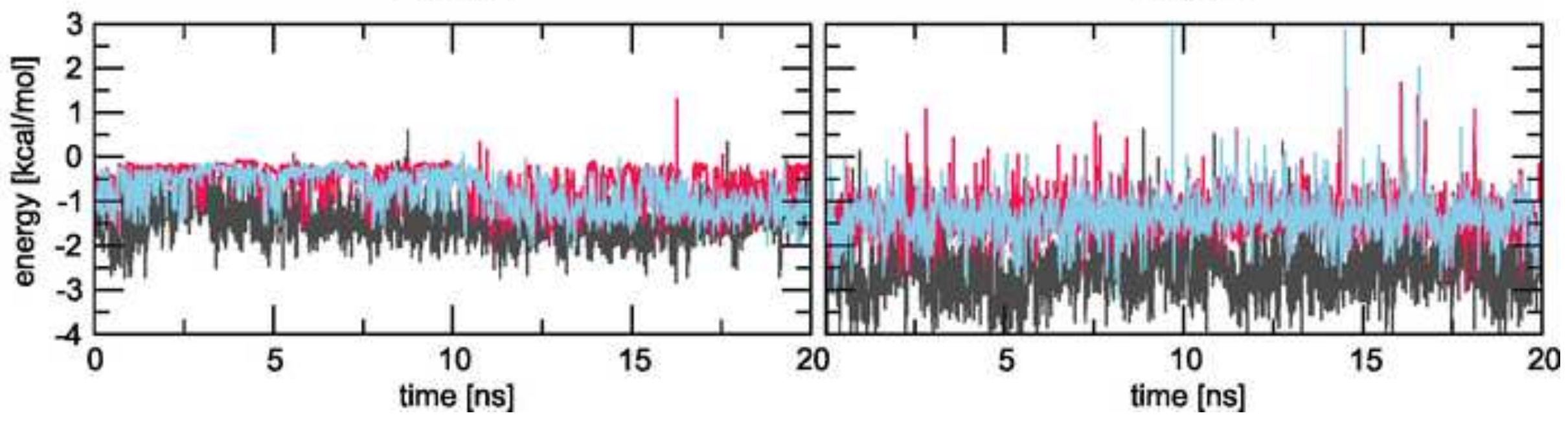

